\title{
Complementary role of the pressure in the black hole thermodynamics
}

\author{
Edwin J. Son ${ }^{1, *}$ and Wontae $\mathrm{Kim}^{2,3,+}$ \\ ${ }^{1}$ Division of Computational Mathematics, \\ National Institute for Mathematical Sciences, \\ Daejeon 305-390, Republic of Korea \\ ${ }^{2}$ Department of Physics, Sogang University, Seoul 121-742, Republic of Korea \\ ${ }^{3}$ Center for Quantum Spacetime, Sogang University, Seoul 121-742, Republic of Korea
}

(Dated: November 3, 2018)

\begin{abstract}
In black hole thermodynamics of certain models, the thermodynamic first law may contain the pressure term. The corresponding entropy follows the area law whereas the thermodynamic energy is not the same with the black hole mass. If the pressure can be decomposed into two parts and recombined with the original thermodynamic quantities, then the thermodynamic energy becomes the black hole mass and the entropy satisfying the area law turns out to be the corrected entropy called the Wald entropy, respectively.

PACS numbers: 04.70.Dy, 04.62.+v
\end{abstract}

Keywords: Black Hole, Thermodynamics

*eddy@nims.re.kr

wtkim@sogang.ac.kr 
Since the thermodynamic first law for black holes has been extensively used in black hole thermodynamics and phase transitions ${ }_{1}[4]$, it has been claimed that the first law is essentially related to the Einstein equation [5]. Recently, the remarkable similarity between them has been emphasized by investigating it at the horizon [6]. Explicitly, it shows that the Einstein tensor $G_{r}^{r}$ for spherically symmetric static black holes can be decomposed into two parts; the first part is of relevance to the energy, while the second part is associated with the entropy as long as the Hawking temperature is identified with the surface gravity. Additionally, if there is a source terms for matter, then the pressure of $P=T_{r}^{r}$ from energymomentum tensors is included in the thermodynamic first law. After all, it becomes the thermodynamic first law, $d E-T_{H} d S=-P d V$, where $T_{H}$ is the Hawking temperature and $V$ is the volume of the black hole. Subsequently, we can easily see that what the energy $E$ and the entropy $S$ for the black hole are from this relation. Furthermore, the relationship between the first law of thermodynamics and gravitational field equations in a spherically symmetric space-time has been extensively investigated in the modified gravity theories so that the thermodynamic quantities obtained from the gravitational field equations are consistent with those from other approaches [7 9]. In particular, the thermodynamic energy of a spherically symmetric black hole in a Lorentz noninvariant model has shown to be exactly same with a generalization of the Misner-Sharp energy [9].

However, the pressure can be sometimes regarded as a part of the geometric part such as the Einstein tensor instead of the source. It means that the pressure can be split into two parts and recombined with the original energy and the entropy, which yield modified ones. There are at least two kinds of representations depending on whether the pressure is kept in the right-hand side of the equation or not. In this paper, we would like to apply the above two different approaches to two special models so that we show that the thermodynamic energy can become the black hole mass and the entropy can turn out to be the Wald entropy [10] if the pressure is decomposed and recombined with the original energy and the pressure.

The first illustration is the four-dimensional exact soluble model [11] in the semiclassical Einstein equation with the conformal anomaly [12, 13],

$$
G_{\mu \nu}=8 \pi G_{N}\left\langle T_{\mu \nu}\right\rangle
$$

where $G_{\mu \nu}=R_{\mu \nu}-\frac{1}{2} g_{\mu \nu} R$ and $\left\langle T_{\mu \nu}\right\rangle$ is the effective energy-momentum tensor from the trace anomaly, $g^{\mu \nu}\left\langle T_{\mu \nu}\right\rangle=-\alpha\left(8 \pi G_{N}\right)^{-1} E_{(4)}+\tilde{\alpha} C_{\alpha \beta \kappa \lambda} C^{\alpha \beta \kappa \lambda}$, where the coefficients $\alpha$ and $\tilde{\alpha}$ 
depend on matter contents, $E_{(4)}=R_{\mu \nu \kappa \lambda} R^{\mu \nu \kappa \lambda}-4 R_{\mu \nu} R^{\mu \nu}+R^{2}$ is the Gauss-Bonnet term, and $C_{\alpha \beta \kappa \lambda}$ is the Weyl tensor. By assuming $\tilde{\alpha}=0$ and $\alpha>0$ for the exact solubility with the relations of $\left\langle T_{t}^{t}\right\rangle=\left\langle T_{r}^{r}\right\rangle$ in the whole space-time [11], one can find the asymptotic flat exact solution, $d s^{2}=-f(r) d t^{2}+f^{-1}(r) d r^{2}+r^{2} d \Omega_{2}^{2}$,

$$
f(r)=1-\frac{r^{2}}{4 \alpha}\left[1-\sqrt{1-\frac{16 \alpha G_{N} M}{r^{3}}-\frac{4 \alpha a^{2}}{r^{4}}}\right],
$$

where $M$ and $a$ are integration constants. The metric (2) has two roots satisfying $f\left(r_{ \pm}\right)=0$; however, the horizon is obtained as $r_{+}=G_{N} M+\sqrt{G_{N}^{2} M^{2}+2 \alpha}$ because $r_{-}$becomes negative for $\alpha>0$. Following the procedure in Ref. [6], the relevant tensor component for the static spherically symmetric solution (2) is given by only the radial part,

$$
G_{r}^{r}=8 \pi G_{N}\left\langle T_{r}^{r}\right\rangle
$$

where the Einstein tensor and the anomaly term can be written as $G_{r}^{r}(r)=-r^{-2}\left(1-f-r f^{\prime}\right)$, $\left\langle T_{r}^{r}\right\rangle=\alpha\left(4 \pi G_{N}\right)^{-1} r^{-4}(1-f)\left(1-f+2 r f^{\prime}\right)$, respectively. The prime means the derivative with respect to the radial coordinate. Considering Eq. (3) at the event horizon of $r=r_{+}$, one can obtain the equation of motion,

$$
d\left(\frac{r_{+}}{2 G_{N}}\right)-\frac{f^{\prime}\left(r_{+}\right)}{4 \pi} d\left(\frac{\pi r_{+}^{2}}{4 G_{N}}\right)=-\left.\left\langle T_{r}^{r}\right\rangle d V\right|_{r=r_{+}},
$$

where $V$ is the volume of the black hole. Now, the Hawking temperature is defined by the surface gravity of $T_{\mathrm{H}}=f^{\prime}\left(r_{+}\right) / 4 \pi$ and the pressure is given by $P=\left\langle T_{r}^{r}\right\rangle$. Requiring the thermodynamic first law,

$$
d \tilde{E}-T_{H} d \tilde{S}=-\left.P d V\right|_{r=r_{+}}
$$

we obtain the thermodynamic energy and the entropy as

$$
\tilde{E}=\frac{r_{+}}{2 G_{N}}, \quad \tilde{S}=\frac{\pi r_{+}^{2}}{G_{N}}=\frac{A}{4 G_{N}},
$$

where $A=4 \pi r_{+}^{2}$. Note that the thermodynamic energy is not the same with black hole mass, while the entropy satisfies the well-known area law in the presence of pressure. On the other hand, the above quantum-mechanically induced pressure can be decomposed into two parts and they are absorbed into the energy and the entropy, so that Eq. (4) can be written as

$$
d\left(\frac{r_{+}}{2 G_{N}}-\frac{\alpha}{G_{N} r_{+}}\right)-\frac{f^{\prime}\left(r_{+}\right)}{4 \pi} d\left(\frac{A}{4 G_{N}}-\frac{4 \pi \alpha}{G_{N}} \ln \frac{A}{A_{0}}\right)=0 .
$$


Note that there appears the $\alpha$ correction at each term due to the anomaly. Comparing Eq. (7) with the thermodynamic first law of $d E-T_{\mathrm{H}} d S=0$, we can identify the modified thermodynamic energy and the modified entropy as

$$
E=M, \quad S=\frac{A}{4 G_{N}}-\frac{4 \pi \alpha}{G_{N}} \ln \frac{A}{A_{0}} .
$$

Note that the energy is nothing but the black hole mass which can be compactly written in terms of the inverse relation of the event horizon, $M=r_{+} / 2-\alpha / r_{+}$, and the constant $A_{0}$ reflects the logarithmic ambiguity. The entropy receives the logarithmic correction which is the same with the Wald entropy in Ref. [11] (another approach can be found in Ref. [14]).

Second, let us start with the $d$-dimensional Einstein-Hilbert action $I_{\mathrm{EH}}$ coupled to a Gauss-Bonnet term $I_{1}$ with a negative cosmological constant $\Lambda=-(d-1)(d-2) / 2 \ell^{2}$,

$$
\begin{aligned}
I & =I_{\mathrm{EH}}+I_{1} \\
& =\frac{1}{16 \pi G_{N}} \int d^{d} x \sqrt{-g} R+\frac{1}{16 \pi G_{N}} \int d^{d} x \sqrt{-g}\left[-2 \Lambda+\frac{\alpha}{(d-3)(d-4)} E_{(4)}\right],
\end{aligned}
$$

where $d$ is considered greater than four. Variation of the action (9) yields the equations of motion,

$$
\begin{aligned}
G_{\mu \nu}=8 \pi G_{N} T_{\mu \nu} & \\
\equiv-\Lambda g_{\mu \nu}-\frac{\alpha}{(d-3)(d-4)}\left[2 R R_{\mu \nu}\right. & -4 R_{\mu \kappa} R_{\nu}^{\kappa}-4 R^{\kappa \lambda} R_{\mu \kappa \nu \lambda} \\
& \left.+R_{\mu \kappa \lambda \sigma} R_{\nu}^{\kappa \lambda \sigma}-\frac{1}{2} g_{\mu \nu} E_{(4)}\right],
\end{aligned}
$$

where $T_{\mu \nu}=-\left(8 \pi G_{N}\right)^{-1} \delta I_{1} / \delta g^{\mu \nu}$. The metric is assumed to be the static metric of $d s^{2}=$ $-f(r) d t^{2}+f^{-1}(r) d r^{2}+r^{2} d \Omega_{(d-2), k}^{2}$, where $d \Omega_{(d-2), k}^{2}$ is the line element of a $(d-2)$-dimensional totally symmetric space with a constant curvature $(d-2)(d-3) k$. Then, the explicit form of the solution is given as [15],

$$
f(r)=k+\frac{r^{2}}{2 \alpha}\left[1 \pm \sqrt{1+\frac{64 \pi G_{N} \alpha M}{(d-2) \Sigma_{k} r^{d-1}}-\frac{4 \alpha^{2}}{\ell^{2}}}\right]
$$

where $\Sigma_{k}$ is the volume of the $(d-2)$-dimensional space. There are two branches so that we take the $(-)$ sign which goes to the Schwarzschild metric asymptotically. Then the mass can be written in terms of the horizon $r_{+}$as

$$
M=\frac{(d-2) \Sigma_{k} r_{+}^{d-3}}{16 \pi G_{N}}\left[k+\frac{r_{+}^{2}}{\ell^{2}}+\frac{\alpha k^{2}}{r_{+}^{2}}\right],
$$


where the horizon satisfies $f\left(r_{+}\right)=0$. Using the metric, the relevant Einstein tensor and the pressure from the energy-momentum tensors can be calculated as

$$
\begin{aligned}
G_{r}^{r} & =\frac{(d-2)}{2}\left[\frac{f^{\prime}(r)}{r}-\frac{(d-3)(k-f(r))}{r^{2}}\right] \\
T_{r}^{r} & =-\frac{(d-2) \alpha}{8 \pi G_{N}}\left[\frac{f^{\prime}(r)(k-f(r))}{r^{3}}+\frac{(d-5)}{2 r^{4}}\left(f(r)(2 k-f(r))-k^{2}\right)\right]+\frac{(d-1)(d-2)}{16 \pi G_{N} \ell^{2}},
\end{aligned}
$$

respectively, so that the Einstein equation (10) can be written as

$$
\frac{(d-2)(d-3) \Sigma_{k} k r_{+}^{d-4}}{16 \pi G_{N}} d r_{+}-\frac{f^{\prime}\left(r_{+}\right)}{4 \pi}\left[\frac{(d-2) \Sigma_{k} r_{+}^{d-3}}{4 G_{N}}\right] d r_{+}=-\left.T_{r}^{r} d V\right|_{r=r_{+}} .
$$

Defining the pressure $P=T_{r}^{r}$ and the differential volume $d V=\Sigma_{k} r^{d-2} d r$, we can regard the Einstein equation with matter as the thermodynamic first law [6],

$$
d \tilde{E}-T_{H} d \tilde{S}=-\left.P d V\right|_{r=r_{+}}
$$

as long as we identify the energy and the entropy with

$$
\tilde{E}=\frac{(d-2) \Sigma_{k} k r_{+}^{d-3}}{16 \pi G_{N}}, \quad \tilde{S}=\frac{A}{4 G_{N}}
$$

where $A=\Sigma_{k} r_{+}^{d-2}$. Note that the thermodynamic energy cannot be written as the black hole mass; in particular, the entropy satisfies the area law. Similar to the first illustration, the pressure in Eq. (14) is transposed to the left-hand side of the Einstein equation and then it can be decomposed into two terms as in the following:

$$
\begin{array}{r}
\frac{(d-2) \Sigma_{k}}{16 \pi G_{N}}\left[(d-3) k r_{+}^{d-4}+\frac{(d-1) r_{+}^{d-2}}{\ell^{2}}+(d-5) \alpha k^{2} r_{+}^{d-6}\right] d r_{+} \\
-\frac{f^{\prime}\left(r_{+}\right)}{4 \pi}\left[\frac{(d-2) \Sigma_{k} r_{+}^{d-3}}{4 G_{N}}\left(1+\frac{2 \alpha k}{r_{+}^{2}}\right)\right] d r_{+}=0 .
\end{array}
$$

Identifying Eq. (18) with the thermodynamic first law of $d E=T_{H} d S$ without the pressure, we can get the modified energy and the modified entropy,

$$
E=M, \quad S=\frac{\Sigma_{k} r_{+}^{d-2}}{4 G_{N}}\left[1+\frac{2(d-2) \alpha k}{(d-4) r_{+}^{2}}\right]
$$

The superficially complicated thermodynamic energy becomes just the black hole mass and the entropy can be written as the Wald entropy which is compatible with the previous result in Ref. [15]. In particular, as seen from Eq. (18), there is no explicit Gauss-Bonnet correction 
to the energy in five dimensions, whereas the mass formula (12) still has an $\alpha$ correction. However, the $\alpha$ correction term in the mass formula is actually independent of the horizon, so that it can be an integration constant. For a spherically symmetric black hole without the cosmological constant, these relations have been obtained in Ref. [7] and are consistent with our results.

In conclusion, we have studied two kinds of expressions of the thermodynamic first law depending on the existence of the pressure throughout the two black hole models. In the presence of the pressure in the thermodynamic law, the entropy of the black hole can be written as the well-known area law, while the thermodynamic energy cannot be written as the black hole mass. In contrast to this expression, if the pressure is eliminated, then the entropy receives some corrections to the standard area law, while the thermodynamic energy of the black hole becomes the black hole mass. So, the black hole mass and the Bekenstein-Hawking entropy seems to be complementary in the presence of the pressure.

\section{Acknowledgments}

This work was supported by the National Research Foundation of Korea(NRF) grant funded by the Korea government (MEST) (2010-0008359).

[1] J. D. Bekenstein, Phys. Rev. D 7, 2333 (1973).

[2] J. D. Bekenstein, Phys. Rev. D 9, 3292 (1974).

[3] S. W. Hawking, Commun. Math. Phys. 43, 199 (1975) [Erratum-ibid. 46, 206 (1976)].

[4] S. W. Hawking and D. N. Page, Commun. Math. Phys. 87, 577 (1983).

[5] T. Jacobson, Phys. Rev. Lett. 75, 1260 (1995) gr-qc/9504004.

[6] T. Padmanabhan, Res. Astron. Astrophys. 12, 891 (2012) [arXiv:1207.0505 [astro-ph.CO]].

[7] A. Paranjape, S. Sarkar and T. Padmanabhan, Phys. Rev. D 74, 104015 (2006) hep-th/0607240].

[8] M. Akbar and R. -G. Cai, Phys. Lett. B 648, 243 (2007) gr-qc/0612089].

[9] R. -G. Cai and N. Ohta, Phys. Rev. D 81, 084061 (2010) [arXiv:0910.2307 [hep-th]].

[10] R. M. Wald, Phys. Rev. D 48, 3427 (1993) gr-qc/9307038. 
[11] R.-G. Cai, L.-M. Cao and N. Ohta, JHEP 1004, 082 (2010) [arXiv:0911.4379 [hep-th]].

[12] S. Deser and A. Schwimmer, Phys. Lett. B 309, 279 (1993) hep-th/9302047.

[13] M. J. Duff, Class. Quant. Grav. 11, 1387 (1994) hep-th/9308075.

[14] R. Banerjee and B. R. Majhi, Phys. Lett. B 674, 218 (2009) [arXiv:0808.3688 [hep-th]].

[15] R.-G. Cai, Phys. Rev. D 65, 084014 (2002) hep-th/0109133. 